\title{
Consciência metalinguística e compreensão de leitura: diferentes facetas de uma relação complexa ${ }^{1}$
}

\section{Metalinguistic awareness and reading comprehension: Different facets of a complex relationship}

\author{
Alina Galvão Spinillo \\ Márcia Maria Peruzzi Elia da Mota ${ }^{3}$ \\ Jane Correa ${ }^{4}$
}

\begin{abstract}
RESUMO
O presente artigo, de natureza teórica e com base em pesquisas realizadas com crianças, discute as complexas relações entre consciência metalinguística e compreensão leitora a partir da consciência fonológica, da consciência morfológica e da consciência metatextual. A consciência fonológica refere-se à habilidade de refletir acerca dos sons que compõem as palavras, auxiliando no processo de decodificação que, por sua vez, contribui para a compreensão de leitura. A consciência morfológica pode ser entendida como a habilidade de refletir sobre os morfemas, que são as menores unidades linguísticas que têm significado próprio. O que se observa é que a informação relativa à decodificação interage com informações sintático-semânticas oriundas do conhecimento que o leitor possui acerca da morfologia da língua, contribuindo para a leitura de textos. A consciência metatextual refere-se à habilidade de refletir sobre as propriedades dos textos. Diferentemente da consciência fonológica e morfológica, as relações entre a consciência
\end{abstract}

1 Agradecemos os apoios a nós concedidos por diferentes agências de fomento (CAPES Coordenação de Aperfeiçoamento de Pessoal de Nível Superior; $\mathrm{CNPq}$ - Conselho Nacional de Desenvolvimento Científico e Tecnológico); FACEPE- Fundação de Amparo à Ciência e Tecnologia do Estado de Pernambuco; FAPERJ- Fundação de Amparo à Pesquisa do Estado do Rio de Janeiro) às pesquisas que contribuíram para a escrita deste trabalho.

2 Doutora em Psicologia pela Universidade de Oxford, Inglaterra; Professora da Universidade Federal de Pernambuco- Brasil. alinaspinillo@hotmail.com

3 Doutora em Psicologia pela Universidade de Oxford, Inglaterra; Professora da Universidade Salgado de Oliveira e da Universidade do Estado do Rio de Janeiro - Brasil. mmotapsi@gmail.com

4 Doutora em Psicologia pela Universidade de Oxford, Inglaterra; Professora da Universidade Federal do Rio de Janeiro - Brasil.jncrrea@gmail.com 
metatextual e a compreensão leitora não são tão claras, havendo resultados divergentes quanto à contribuição do conhecimento sobre as propriedades do texto para a compreensão textual. $\mathrm{O}$ entendimento dessas relações permite construir um quadro teórico a respeito do papel da consciência metalinguística, seja em relação ao fonema, ao morfema ou às propriedades do texto, na compreensão textual; bem como extrair implicações educacionais acerca da aprendizagem da leitura.

Palavras-chave: consciência metalingüística; compreensão de leitura; crianças.

\begin{abstract}
This paper discusses the complex relationship between metalinguistic awareness and reading comprehension, based on phonological, morphological and metatextual awareness. Although it has been based on researches with children, this paper has a theoretical focus. Phonological awareness refers to the ability to reflect on the sounds that form words, supporting the decoding process, which in turn contributes to reading comprehension. Morphological awareness is understood as the ability to reflect on the morphemes of a language, which are the smallest linguistic units that possess meaning. We observe that the information related to decoding interacts with syntacticsemantic information, which stems from the knowledge the reader has about the morphology of the language, contributing to the reading of texts. Metatextual awareness refers to the ability to reflect on texts' properties. Different from phonological and morphological awareness, the relationship between metatextual awareness and reading comprehension are not so clear, and there are diverging results as to the contribution of knowing of a text's properties to the understanding of that text. The understanding of these relations allows us to build a theoretical framework about the role of metalinguistic awareness, whether in relation to the phoneme, the morpheme, or the properties of the text, in reading comprehension, as well as derive from this framework educational implications about the process of learning how to read.
\end{abstract}

Keywords: metalinguistic awareness; reading comprehension; children.

\title{
Introdução
}

Embora tenha como função primária ser um objeto de comunicação, a linguagem pode torna-se, também, objeto de reflexão e análise; tornando-se ela própria o foco de atenção deliberada por parte do indivíduo. Esta atividade, denominada consciência metalinguística, é realizada por um indivíduo que 
trata a linguagem como um objeto cujas características podem ser examinadas a partir de um monitoramento intencional, que exige um distanciamento em relação aos usos da linguagem e uma aproximação da forma em que a linguagem se apresenta. Ao explicitamente focalizar sua atenção na linguagem, o indivíduo torna-se capaz de analisá-la e manipulá-la (GARTON; PRATT, 1998; HERRIMAN, 1986; PRATT; GRIEVE, 1984; SPINILLO, 2009; SPINILLO; SIMÕES, 2003).

A consciência metalinguística se subdivide em diferentes tipos, conforme classificação de Tunmer e Herriman (1984) e de Gombert (1992): consciência fonológica; consciência morfológica; consciência sintática ${ }^{5}$; consciência metatextual e consciência pragmática. Esta classificação leva em conta as instâncias linguísticas que são tomadas pelo indivíduo como foco de sua atenção, a saber: o fonema, a palavra, a sintaxe, o texto e o contexto no qual a linguagem se insere. As três primeiras instâncias são amplamente investigadas por pesquisadores interessados em examinar as relações entre elas e a aprendizagem da escrita pela criança, sobretudo no que tange à consciência fonológica. Como forma de contribuir com novas reflexões a respeito da consciência metalinguística, o presente artigo, de natureza teórica e com base em pesquisas realizadas com crianças no Brasil e no exterior, discute as complexas relações entre consciência metalinguística e compreensão leitora a partir da consciência fonológica, da consciência morfológica e da consciência metatextual. $\mathrm{O}$ entendimento dessas relações contribui para a construção de um quadro teórico mais articulado a respeito do papel da consciência metalinguística na compreensão textual, como também pode contribuir para a construção de práticas educacionais mais apropriadas acerca da compreensão textual em leitores iniciantes, como é o caso de crianças nos anos iniciais do ensino fundamental.

\section{Consciência fonológica e compreensão leitora}

A consciência fonológica diz respeito à habilidade de segmentação, análise e manipulação intencional dos sons que compõem a fala (GOMBERT, 1992), podendo ser entendida como um construto multidimensional, uma vez que é composta por uma constelação de habilidades, como por exemplo, a segmentação, a subtração e julgamento de sílabas ou fonemas (YOPP, 1988). Tais habi-

5 Alguns autores se referem à consciência morfossintática de maneira integrada. 
lidades variam também quanto a sua complexidade, considerada a atividade de análise fonológica requerida, bem como a unidade sonora objeto desta análise (GOUGH; LARSON; YOPP, 1995).

Inúmeras são as evidências acumuladas nos últimos trinta anos acerca da importância da consciência fonológica para o aprendizado da língua escrita (BRADLEY; BRYANT, 1983; GOMBERT, 1992; GUIMARÃES, 2003; PLAZA; COHEN, 2003; SANTOS, 2009). Tais evidências empíricas tomam por base diversas ortografias alfabéticas, incluindo o português brasileiro (CARDOSOMARTINS, 1995a; 1995b; BARRERA; MALUF, 1997). Observa-se, no entanto, que as habilidades de consciência fonológica consideradas importantes para o aprendizado inicial da linguagem escrita podem variar em função da natureza da ortografia aprendida (CARAVOLAS, 1993). As habilidades relacionadas ao julgamento de rima e aliteração, por exemplo, estão associadas ao desenvolvimento da habilidade de leitura em inglês (BRADLEY; BRYANT, 1983), enquanto habilidades relacionadas à consciência silábica estão associadas ao aprendizado do português brasileiro (MOUSINHO; CORREA, 2009).

A relação estabelecida entre consciência fonológica e alfabetização é a de causalidade recíproca (GOMBERT, 2003; GOSWAMI; BRYANT, 1990; WAGNER; TORGESEN, 1987). Certas habilidades de consciência fonológica precedem a alfabetização e auxiliam o processo de aprendizado da linguagem escrita. Este aprendizado, por sua vez, promove o desenvolvimento ulterior de outras habilidades de análise fonológica, especialmente aquelas relacionadas à consciência fonêmica. Estas, por sua vez, favorecem o progresso da criança na leitura e na escrita, e assim sucessivamente.

A influência da consciência fonológica para o aprendizado da linguagem escrita não se aplica apenas ao desenvolvimento típico. O desenvolvimento da consciência fonológica é importante também para o domínio da leitura e da escrita por aprendizes com necessidades educativas especiais (CARDOSOMARTINS; SILVA, 2008). De acordo com várias pesquisas, o treinamento das habilidades de consciência fonológica auxilia a remediação dos problemas de leitura e escrita (BRADLEY; BRYANT, 1985; CAPOVILLA; CAPOVILLA, 2000; CUNINGHAN, 1990; GOSWAMI; BRYANT, 1990).

A consciência fonológica está geralmente associada ao entendimento do princípio alfabético. O princípio é o de que nas línguas alfabéticas as letras (grafemas) correspondem aos sons (fonemas) da fala. A aquisição desse princípio é de grande importância para que se possa aprender a ler e escrever línguas que possuem este tipo de ortografia. Neste sentido, a consciência fonológica auxilia o processo de decodificação de palavras realizado no ato de ler. Por sua vez, a habilidade de decodificação contribui para a compreensão de leitura (SALLES; PARENTE, 2002; TUNMER; HOOVER, 1992; YUILL; OAKHILL, 1991). A 
automatização do processo de decodificação, ao acelerar o reconhecimento das palavras, libera espaço de memória para o armazenamento do texto, facilitando, assim, o processo de compreensão (STANOVICH, 1980).

Para a leitura concorrem outras habilidades linguístico-cognitivas além daquelas associadas à consciência fonológica. Durante a leitura, informações contextuais podem também ser empregadas pelas crianças juntamente com pistas grafofonêmicas (TUNMER; HOOVER, 1992). Assim, quando não reconhece uma palavra na frase, a criança pode empregar a decodificação parcial da palavra associada ao contexto para reconhecê-la, o que favorece a compreensão leitora. Por exemplo, na frase "Vovó toma o ca**", o contexto oferece a informação de que se necessita de um substantivo masculino (uma vez que o artigo " $O$ " define o gênero) e que, ao mesmo tempo, a palavra deve se referir a algo que possa ser bebido. A decodificação parcial da sílaba " $c a$ ", por sua vez, indica que somente bebidas que começam com este som $/ \mathrm{ka} /$ completariam a palavra e, consequentemente, a frase. Desta forma, o contexto auxiliaria também a criança no aprendizado de novas correspondências grafofonêmicas.

Embora de contribuição inegável, o emprego de informações contextuais é superestimado na perspectiva segundo a qual a leitura poderia prescindir da decodificação em favor da utilização de tais informações (GOODMAN, 1982). Segundo esta perspectiva, a criança empregaria seus conhecimentos linguísticos para compreender o texto sem a necessidade de decodificar as palavras. Smith (1971) observa que muito da memória de trabalho da criança que começa a aprender a ler é ocupada pela lentidão com que o processo de decodificação ocorre. Desta forma, os recursos cognitivos depreendidos na decodificação dificultariam o processamento de outras informações contidas no texto, o que se tornaria, desta forma, um obstáculo para a sua compreensão. A realização da leitura por meio da decodificação das palavras tornaria o processo de compreensão do texto custoso e enfadonho para a criança. Por não depender da decodificação, na leitura contextual, a compreensão do texto ficaria facilitada, pois o reconhecimento das palavras seria realizado por meio da formação de um vocabulário visual.

Ao contrário do que pensavam Goodman (1982) e Smith (1971), a decodificação eficiente é uma habilidade importante para o aprendizado e desenvolvimento da leitura, conforme demonstrado em estudos posteriores como os de Nicholson (1993), Tunmer (1990) e Stanovich (1981). Embora pistas contextuais sejam utilizadas, os leitores com maior facilidade de compreensão de leitura são também os que possuem melhor habilidade de decodificação. Leitores habilidosos não são tão dependentes do contexto, pois decodificam bem as palavras; ao contrário de leitores principiantes e leitores menos habilidosos que compensam suas dificuldades em decodificação dependendo mais das pistas contextuais 
(STANOVICH, 1980). Neste sentido, observa-se que leitores habilidosos têm melhor desempenho do que seus pares menos habilidosos em tarefas relacionadas ao processamento fonológico (SILVA; MOTA, 2009); o mesmo acontece quando comparados leitores e não leitores (MOUSINHO; CORREA, 2009).

Gough e Tumner (1986) apresentaram um modelo simples para a leitura envolvendo a relação multiplicativa entre a decodificação e a compreensão oral (leitura $=$ decodificação x compreensão). De acordo com o modelo proposto (HOOVER; GOUGH, 1990), o desenvolvimento da leitura não ocorreria na ausência de qualquer das duas habilidades mencionadas no modelo. Dificuldades encontradas pela criança na decodificação trariam, como consequência imediata, dificuldades na compreensão leitora. Por outro lado, dificuldades na compreensão leitora poderiam ser observadas na ausência de dificuldades na habilidade de decodificação. Neste sentido, a leitura como atividade de produção de sentido tem como fundamento necessário, embora não suficiente, o desenvolvimento de habilidades metacognitivas de processamento fonológico que possam tornar possível a ocorrência da decodificação no ato de ler.

\section{Consciência morfológica e compreensão leitora}

A consciência fonológica não é a única habilidade que facilita o processo de aquisição da leitura e da escrita. Outra habilidade metalinguística que tem se mostrado relevante é a consciência morfológica (CARLISLE, 1988, 1995, 1996, 2000; DEACON; KIRBY, 2004; NAGY; BERNINGER; ABBOT, 2006). Consciência morfológica é a habilidade de refletir sobre os morfemas, menores unidades linguísticas que tem significado próprio (CARLISLE, 1995). Essa habilidade pode ajudar na compreensão de leitura porque a ortografia de muitas palavras depende da morfologia. Palavras como "laranjeira", que tem ortografia ambígua (com $g$ ou $\operatorname{com} j$ ), podem ser escritas de forma correta se a criança souber sua origem: "laranja". Os significados dessas palavras podem ser igualmente inferidos na leitura, se o leitor souber o significado da palavra que as originou. No caso particular da leitura contextual, pode-se ressaltar que tanto os aspectos ligados à decodificação, que foram destacados na seção anterior, como também aspectos sintáticos e semânticos afetam a compreensão do texto.

Em um estudo que pretendia explorar essa questão no português do Brasil, Mota et al. (2009) investigaram a relação entre o processamento 
fonológico (tarefa de dígitos) e a consciência morfológica em crianças de segundo e terceiro ano do ensino fundamental. As crianças realizaram tarefas de consciência morfológica. A compreensão de leitura foi medida pelo teste de Cloze e o processamento fonológico pela tarefa de dígitos do WISC. Os resultados mostraram que o processamento fonológico contribuiu para a compreensão leitora medida pelo teste de Cloze. Necessário lembrar que Tunmer (1990) considera que um importante aspecto da compreensão de leitura é também a utilização de pistas grafofonêmicas associadas ao uso de informações contextuais, como no exemplo anteriormente apresentado em relação à palavra "café". Assim, os resultados podem indicar que a hipótese de Tunmer é correta. Isto é, algum tipo de processamento fonológico ocorre na leitura contextual, provavelmente quando a criança parcialmente decodifica as palavras.

Quanto à consciência morfológica, os dados parecem corroborar a ideia de que essa informação relativa à decodificação interage com informações sintático-semânticas oferecidas pelo conhecimento da morfologia da língua. A relação entre o processamento da morfologia e a leitura contextual foi demonstrada pelas correlações positivas e significativas entre a medida de leitura e as medidas de consciência morfológica. Desta forma, as crianças que tiveram maiores escores no teste de Cloze foram também aquelas com maiores escores nas tarefas de consciência morfológica.

Esses resultados sugerem que além do processamento fonológico, o processamento morfológico contribui para a leitura contextual. Embora correlações não estabeleçam relações de causa e efeito, esses resultados indicam que essa questão deve ser investigada em estudos que possam estabelecer se há, de fato, uma relação causal entre essas variáveis.

\section{Consciência metatextual e compreensão de leitura}

Nas seções anteriores, a compreensão de leitura era tratada a partir da leitura de palavras, uma vez que eram os aspectos fonológicos e morfolólogicos que eram o foco da discussão. Ao focalizar agora a consciência metatextual, a compreensão é tratada a partir da leitura de textos.

Comparada com a consciência fonológica e com a consciência morfológica, a relação entre consciência metatextual e compreensão de leitura é tema pouco explorado. Isso se justifica pelo fato de que o termo consciência metatextual é relativamente recente na literatura, havendo sido cunhado por Gombert 
$(1992)^{6}$; e pelo fato de que investigações sobre este tema ainda são raras, sendo mais raro ainda o exame das relações entre esta consciência e outras habilidades linguísticas como a compreensão de textos.

Revisando-se a literatura na área (SPINILLO, 2009; SPINILLO; SIMÕES, 2003), é possível observar que, ainda que de forma indireta, algumas investigações exploraram a possível relação entre a consciência metatextual e a compreensão de textos, como é o caso das pesquisas conduzidas por Ruffman (1996), por Cain e Oakhill (1996) e por Simões (2002); cada uma delas investigando um aspecto distinto desta relação.

O estudo de Ruffman (1996) voltava-se para o monitoramento da compreensão, ou seja, a capacidade do leitor de tomar consciência de que está ou não compreendendo o que está lendo. Assim como outros estudos acerca do monitoramento, esta pesquisa examinava a habilidade de crianças em detectar informações contraditórias ${ }^{7}$ em textos que lhes eram apresentados. $\mathrm{O}$ autor enfatiza a importância da capacidade do leitor em detectar erros e inconsistências em um texto e a capacidade de tomar consciência de que essas inconsistências dificultam sua compreensão; visto que leitores iniciantes ou com dificuldades de compreensão têm dificuldade em monitorar sua leitura, sendo este resultado também observado por Yuill e Oakhill (1991). Considerando o monitoramento como uma atividade metatextual, no sentido de tomar o texto como objeto de reflexão e análise, os resultados do estudo de Ruffman sugerem haver, portanto, uma relação entre a capacidade da criança de compreender textos e a capacidade de monitorar sua leitura.

Cain e Oakhill (1996) exploraram as relações entre compreensão de textos e o conhecimento que crianças possuem sobre história. Crianças de 7 e 8 anos de idade foram divididas em três grupos: crianças com nível de compreensão abaixo do esperado naquela idade, crianças com nível de compreensão acima do esperado naquela idade e crianças com nível de compreensão esperado naquela idade. Todos os participantes foram solicitados a responder perguntas a respeito da natureza ficcional da história, acerca da função do título; acerca da função do início, meio e final da história. As crianças com dificuldades de compreensão apresentavam uma noção limitada acerca de todos esses aspectos, enquanto que aquelas com um bom nível de compreensão sabiam a respeito da natureza ficcional do conteúdo tratado em histórias, reconheciam que o título tinha por

6 A obra original foi publicada em francês em 1990.

7 Em pesquisas sobre monitoramento, as inconsistências podem ser de natureza lógica, em que passagens contraditórias entre si estão presentes no interior do texto, ou podem ser de natureza empírica, em que informações veiculadas no texto divergem do conhecimento de mundo do leitor. 
função anunciar o tema e/ou os personagens presentes em uma história e tinham noções a respeito dos marcadores de abertura e fechamento de histórias. Segundo as autoras, as crianças com dificuldades de compreensão não formam uma representação mental geral do texto que as auxiliem a integrar os episódios entre si e que esta representação, por sua vez, depende do conhecimento que as crianças têm sobre histórias, estabelecendo-se, assim, a relação entre esses fenômenos linguísticos via a capacidade de construir esta representação global.

O estudo de Ruffman (1996) versa sobre o conteúdo do texto, as informações nele veiculadas, enquanto a pesquisa de Cain e Oakhill (1996) versa sobre o conhecimento acerca de aspectos relativos à configuração e organização do texto, no caso sua macroestrutura. Entretanto, é possível dizer que ambos tratavam a consciência metatextual de forma indireta, inclusive sem sequer mencionar este termo em suas pesquisas. É em pesquisas recentes desenvolvidas no Brasil que a consciência metatextual é tratada de forma mais específica, através de tarefas particularmente voltadas para o exame da capacidade da criança de tomar o texto como objeto de reflexão e análise, como é o caso das pesquisas discutidas a seguir.

As relações entre compreensão de texto e consciência metatextual foram examinadas por Simões (2002) em um estudo longitudinal iniciado quando as crianças tinham 7 anos de idade e concluído um ano depois. Em duas ocasiões de testagem elas foram avaliadas em uma tarefa de compreensão de textos e em uma tarefa de consciência metatextual. A tarefa de compreensão de textos consistia em responder perguntas literais e inferenciais sobre uma história lida pela examinadora. A tarefa de consciência metatextual requeria determinar se uma história lida estava completa ou incompleta e identificar, no caso de histórias incompletas, as partes presentes (início, meio e final). Não foram observadas correlações entre consciência metatextual e compreensão de texto, quer na primeira, quer na segunda ocasião de testagem, indicando que essas habilidades são independentes.

Comparando-se o estudo de Cain e Oakhill (1996) e o de Simões (2002), nota-se que embora as tarefas de compreensão de textos nos dois estudos fossem semelhantes, havia diferenças na maneira de avaliar a consciência metatextual nas duas investigações. Na pesquisa de Cain e Oakhill, avaliava-se um conhecimento textual que se referia muito mais a uma representação mental global do texto do que a um conhecimento específico sobre sua estrutura, como ocorria no estudo de Simões. Pode-se dizer que este conhecimento geral apresenta correlação com a compreensão de textos porque ambas as habilidades se apoiam em uma representação mental global do texto. Porém, o conhecimento metalinguístico específico (estrutura do texto) não se correlaciona com a compreensão. Esta é uma possível explicação a respeito da controvérsia entre os resultados desses estudos, explicação que precisa ser mais examinada em pesquisas futuras. Este é um campo de investi- 
gação promissor, visto que, como se percebe, as relações entre consciência metatextual e compreensão de textos parecem ser complexas, porém relevantes.

\section{Comentários finais}

A consciência metalinguística associa-se à habilidade de compreensão de textos a partir de diferentes instâncias: fonema, morfologia e consciência sobre o texto. No que se refere ao fonema e ao morfema, as relações são evidentes e inquestionáveis no sentido de que a consciência fonológica e a consciência morfológica auxiliam no reconhecimento de palavras durante a leitura, de modo que o leitor associa seu conhecimento sobre o fonema e sobre o morfema a pistas contextuais fornecidas por outras palavras e sentenças contidas no texto. Por outro lado, as relações entre a consciência metatextual e a compreensão de textos não são tão claras e evidentes assim. Porém, os estudos, ainda que raros, apontam dados interessantes a respeito desta relação. Embora os dados não indiquem haver uma correlação entre conhecimento sobre a estrutura do texto e habilidade de compreensão, as pesquisas mostram haver uma relação entre monitoramento e compreensão de textos e entre o conhecimento geral sobre textos e compreensão de textos.

De posse dessas informações, dois comentários podem ser feitos: um relativo a possíveis implicações educacionais derivadas das discussões aqui levantadas, e outro relativo a pesquisas futuras que possam esclarecer outros aspectos a respeito das relações entre consciência metalinguística e compreensão de textos.

Do ponto de vista educacional, parece ser evidente a importância de se desenvolver nos alunos do ensino fundamental, que iniciam a aprendizagem da leitura, uma consciência metalinguística, seja em termos de capacidade de refletir sobre os fonemas de modo a, apoiado no contexto, ser capaz de ler palavras e atribuir-lhes significados; seja em termos de uma consciência a respeito dos morfemas que constituem uma palavra sendo capaz de, ao identificar a palavra que a originou (ou o seu radical, por exemplo), fazer generalizações que permitam ler mais palavras do que aquelas com as quais já tem familiaridade. Essas são, sem dúvida, aquisições relevantes que precisam ser valorizadas e exploradas pela escola a partir de situações em que a criança seja solicitada a tomar os fonemas e os morfemas como objeto de reflexão e análise.

De forma análoga, o texto também pode ser objeto de uma análise metalinguística por parte das crianças, sendo proporcionadas situações de monitoramento 
em que o leitor é solicitado a detectar no texto o que não compreendeu durante a leitura ou a identificar inconsistências no texto (entre passagens incongruentes ou entre passagens e o conhecimento de mundo do leitor). Além disso, a escola pode desenvolver nos alunos um conhecimento sobre textos quanto a algumas de suas peculiaridades, como suas convenções linguísticas (abertura e fechamento de histórias), natureza do conteúdo veiculado etc. Em outras palavras, a escola poderia atentar para a contribuição da consciência metalinguística para a compreensão de leitura, propondo situações em que o texto, em todas as suas dimensões, fosse tratado metalinguisticamente.

No entanto, apesar de existirem relações entre essas duas instâncias, a compreensão de textos não pode ser entendida como uma consequência direta da alfabetização, no sentido de que uma vez alfabetizada a criança passa automaticamente a ter um bom nível de compreensão de leitura. Na realidade, há um caminho que não se conclui com a alfabetização e que precisa ser percorrido para que a criança se transforme em um leitor competente. A escola precisaria dar continuidade ao ensino da leitura, tornando a compreensão de textos um objeto de ensino a ser tratado didaticamente.

Do ponto de vista da pesquisa na área, ainda é necessário que sejam conduzidas investigações que esclareçam melhor as relações entre consciência metatextual e compreensão de leitura dada a natureza multifacetada desta relação e por ser uma questão ainda pouco investigada na literatura, tornando-se um desafio a ser assumido por pesquisadores da área.

\section{REFERÊNCIAS}

BARRERA, S.; MALUF, M. R. Consciência metalinguística e alfabetização: um estudo com crianças da primeira série do ensino fundamental. Revista Psicologia: Reflexão e Crítica, v. 16, n. 3, p. 491-502, 1997.

BRADLEY, L.; BRYANT, P. Categorizing sounds and learning to read: a causal connection. Nature, n. 301, p. 419-421, 1983.

. Children's reading problem. Oxford: Basil Blackwells, 1985.

CAIN, K.; OAKHILL, J. The nature of the relationship between comprehension skill and the ability to tell a story. British Journal of Developmental Psychology, n. 14, p. 187-201, 1996. 
CAPOVILLA, A.; CAPOVILLA, F. Efeitos do treino de consciência fonológica em crianças com baixo nível sócio-econômico. Psicologia: Reflexão e Crítica, v. 13, n. 1, p. 7-24, 2000.

CARAVOLAS, M. Language-specific influences of phonology and orthography on emergent literacy. In: ALTARRIBA, J. (Org.). Cognition and culture: a crosscultural approach to cognitive psychology. North-Holland: Elsevier Science Publishers, 1993. p. 177-205.

CARDOSO-MARTINS, C. Consciência fonológica e alfabetização. Petrópolis: Vozes. 1995a.

. Sensitivity to rhymes, syllables, and phonemes in literacy acquisition in Portuguese. Reading Research Quarterly, n. 30, p. 808-828, 1995 b.

CARDOSO-MARTINS, C.; SILVA, J. R. A relação entre o processamento fonológico e a habilidade de leitura: evidência da síndrome de Down e da sídrome de Williams. Psicologia: Reflexão e Crítica, n. 21, p. 83-90, 2008.

CARLISLE, J. An exploratory study of morphological errors in children's written stories. Reading and Writing: An Interdisciplinary Journal, n. 8, p. 61-72, 1996.

. Awareness of the structure and meaning of morphologically complex words: impact on reading. Reading and Writing: An Interdisciplinary Journal, n. 12, p. 169-190, 2000.

. Knowledge of derivational morphology and spelling ability in fourth, six, and eight graders. Applied Psycholinguistics, n. 9, p. 247-266, 1988.

Morphological awareness and early reading achievement. In: FELDMAN, L. (Org.). Morphological aspects of language processing. Hillsdale, NJ: Lawrence Erlbaum, 1995. p. 189-211.

CUNINGHAM, A. E. Implicit versus explicit instruction in phonemic awareness. Journal of Experimental Child Psychology, n. 50, p. 429-444, 1990.

DEACON, S.; KIRBY, J. Morphological awareness: just "more phonological”? The roles of morphological and phonological awareness in reading development. Applied Psycholinguistics, n. 25, p. 223-238, 2004.

GARTON, A.; PRATT, C. Learning to be literate: the development of spoken and written language. Oxford: Blackwell Publishers, 1998. 
GOMBERT, J. E. Atividades metalinguísticas e aprendizagem da leitura. In: MALUF, M. R. (Org.). Metalinguagem e aquisição da escrita: contribuições da pesquisa para a prática da alfabetização. São Paulo: Casa do Psicólogo, 2003. p. 19-64.

1992.

. Metalinguistic Development. Hertfordshire: Harverster Wheatsheaf,

GOODMAN, K. Miscues windows on the reading processes. In: GOLLASCH, F. V. (Org.). Language and literacy: the selected writings of Kenneth S. Goodman. Boston: Routledge \& Kegan Paul, 1982.

GOSWAMI, U.; BRYANT, P. Phonological Skills and Learning to Read. London: Lawrence Erlbaun Associates, 1990.

GOUGH, P. B.; LARSON, K.; YOPP, H. A estrutura da consciência fonológica. In: CARDOSO-MARTINS, C. (Org.). Consciência fonológica e alfabetização. Petrópolis: Vozes, 1995. p. 13-35.

GOUGH, P. B.; TUNMER, W. E. Decoding, reading and reading disability. Remedial and Special Education, n. 7, p. 6-10, 1986.

GUIMARÃES, S. R. K. Dificuldades no desenvolvimento da lectoescrita: O papel das habilidades metalingüísticas. Psicologia: Teoria e Pesquisa, v. 19, n. 1, p. 33-45, 2003.

HERRIMAN, M. L. Metalinguistic awareness and the growth of literacy. In: CASTELL, S.; LUKE, A.; EGAN, K. (Orgs.). Literacy, society and schooling. Cambridge: Cambridge University Press, 1986. p. 159-174.

HOOVER, W. A.; GOUGH, P. B. The simple view of reading. Reading and Writing: An Interdisciplinary Journal, n. 2, p. 127-160, 1990.

MOTA, M; LISBOA, R.; DIAS, J.; GONTIJO, R.; PAIVA, N.; MANSURLISBOA, S.; SILVA, D.; SANTOS, A. Relação entre consciência morfológica e leitura contextual medida pelo teste de Cloze. Psicologia: Reflexão e Crítica, v. 22, n. 2, p. 223-229, 2009. [on-line].

MOUSINHO, R.; CORREA, J. Habilidades linguístico-cognitivas em leitores e não-leitores. Pró-Fono R. Atual. Cient., n. 21, p. 13-118, 2009. 
NAGY, W.; BERNINGER, V.; ABBOT, R. Contributions of morphology beyond phonology to literacy outcome of upper elementary and middle-school students. Journal of Educational Psychology, v. 98, n. 1, p. 134-147, 2006.

NICHOLSON, T. The case against context. In: THOMPSON, G.; TUNMER, W.; NICHOLSON, T. (Orgs.). Reading acquisition processes. Clevedon: Multilingual Matters, 1993. p. 91-103.

PLAZA, M.; COHEN, H. The interaction between phonological processing, syntactic awareness, and naming speed in the reading and spelling performance of first-grade children. Brain and Cognition, n. 53, p. 257-292, 2003.

PRATT, C.; GRIEVE, R. The development of metalinguistic awareness: an introduction. In: TUNMER, W. E.; PRATT, C.; HERRIMAN, M. L. (Orgs.). Metalinguistic awareness in children: theory, research and implications. New York: Springer-Verlag, 1984. p. 2-11.

RUFFMAN, T. Reassessing children's comprehension-monitoring skills. In: CORNOLDI, C.; OAKHILL, J. (Orgs.). Reading comprehension difficulties: Processes and intervention. Mahwah, N. J: Lawrence Erlbaum Associates, 1996. p. 33-67.

SALLES, J. F.; PARENTE, M. A. M. P. Processos cognitivos na leitura de palavras em crianças: relações com compreensão e tempo de leitura. Psicologia: Reflexão e Crítica, v. 15, n. 2, p. 321-331, 2002.

SANTOS, A. A. A. A influência da consciência fonológica na aquisição da leitura e da escrita. In: SISTO, F. F.; OLIVEIRA, G. C.; FINI, L. D. T.; SOUZA, M. T. C. C.; BRENELLI, R. P. (Orgs.). Atuação psicopedagógica e aprendizagem escolar. Petrópolis: Vozes, 1996. p. 213-247.

SILVA, D. A.; MOTA, M. M. P. E. Déficits nas habilidades metalinguísticas em crianças com dificuldades na leitura. Psicologia em Pesquisa (UFJF), v. 3, n. 2, p. 110-118, 2009.

SIMÕES, P. M. U. O desenvolvimento da consciência metatextual e suas relações com a compreensão de histórias. Tese (Doutorado em Psicologia Cognitiva) UFPE, 2002.

SMITH, F. Understanding Reading. New York: Holt, Rinehart and Winston, 1971. 
SPINILLO, A. G. A consciência metatextual. In: MOTA, M. (Org.). Desenvolvimento metalinguístico: questões contemporâneas. São Paulo: Casa do Psicólogo, 2009. p. $77-113$.

SPINILLO, A. G.; SIMÕES, P. O desenvolvimento da consciência metatextual em crianças: questões conceituais, metodológicas e resultados de pesquisas. Psicologia: Reflexão e Crítica, v. 16, n. 3, p. 537-546, 2003.

STANOVICH, K. E. Toward an interactive-compensatory model of individual differences in the development of reading fluency. Reading Research Quarterly, n. 16 , p. 32-71, 1980.

. Relationships between word decoding speed, general name-retrieval ability, and reading progress in first-grade children. Journal of Educational Psychology, v. 73, n. 6, p. 809-815, 1981.

TUNMER, W. The role of language prediction skills in beginning reading. Journal of Educational Studies, New Zealand, v. 25, n. 2, p. 95-112, 1990.

TUNMER, W. E.; HOOVER, W. A. Cognitive and linguistic factors in learning to read. In: GOUGH, P. B.; EHRI, L. C.; TREIMAN, R. (Orgs.). Reading Acquisition. Hillsdale, NJ: Earlbaum, 1992.

TUNMER, W. E.; HERRIMAN, M. L. The development of metalinguistic awareness: a conceptual overview. In: TUNMER, W. E.; PRATT, C.; HERRIMAN, M. L. (Orgs.). Metalinguistic awareness in children: theory, research and implications. Berlin: Springer-Verlag, 1984. p. 12-35.

WAGNER, R. K.; TORGESEN, J. K. The nature of phonological processing and its causal role in the acquisition of reading skills. Psychological Bulletin, n. 101, p. 192-212, 1987.

YOPP, H. The validity of phonemic awareness tests. Reading Research Quarterly, v. 23, n. 2 , p. $159-177,1988$.

YUILL, N.; OAKHILL, J. Children's problems in text comprehension: an experimental investigation. Cambridge: Cambridge University Press, 1991.

Texto recebido em 5 de julho de 2010 .

Texto aprovado em 11 de agosto de 2010 . 